\title{
Viable system model of transnational corporation organizational management based on human-electron analogy
}

\author{
Kun Shi \\ School of Business Administration, \\ Shanghai Lixin University of Accounting and Finance, \\ Songjiang, Shanghai, China \\ E-mail: skyseafc@sina.com
}

\begin{abstract}
Transnational corporations are faced with the organizational management problem in the Internet age because of the complicated economic globalization. Viable system model is introduced to solve the problem as it provides the structure to survive in complex ecological environment and shall make the different organizations viable and living for transnational corporation. In the definition of viable, analogy between human and electron is elaborated and the model proves to be effective for organization innovation of transnational companies.
\end{abstract}

Keywords: Viable System Model; Transnational Corporation; Organization; HumanElectron Analogy.

\section{Introduction}

In the age of Internet, transnational corporations invest in or acquire local partners to shortcut the process of building up local market knowledge. In the trend of economic globalization, there has been an evolution in their roles, responsibilities and expectations of transnational corporations operating in host countries around the world, from exploitive to transactional, then responsive, and finally transformative posture leading broad change. However, traditional theories of organizational management such as " International Structural Stages Model " stated by Stopford and Wells[1] and global matrix organization does not fit the global and specific environment for the corporation[2], so a different way to frame the more complex and subtle array is needed. Bartlett described a transnational organization beyond structure using the model of anatomy, physiology and psychology, redefining the key organization dimension [3]. Inspired by the concept of anatomy, a new idea of model is to be elaborated here based on viable system model while using analogy between human and electron. 


\section{Viable System Model}

The Viable System Model (VSM) is a model of the organizational structure of any viable or autonomous system, in such a way as to meet the demands of surviving in the changing environment. One of the prime features of systems that survive is that they are adaptable to different ecological environment. The VSM expresses a model for a viable system, which is an abstracted cybernetic description that is applicable to any organization that is a viable system and capable of autonomy.

The model was developed by operations research theorist and cybernetician Stafford Beer in his book Brain of the Firm (1972) [4], effectively founded management cybernetics. A viable system is composed of five interacting subsystems which may be mapped onto aspects of organizational structure. In broad terms Systems 1-3 are concerned with the "here and now" of the organization's operations, System 4 is concerned with the "there and then", strategically responses to the effects of external, environmental and future demands on the organization. System 5 is concerned with balancing the "here and now" and the "there and then" to give policy directives which maintain the organization as a viable entity [5].

\section{Human-Electron Analogy}

Human civilization exists in the form of different combinations of cities, towns and villages geographically and socially, recognized as specific nations. Now companies and corporations are the main entities of human economy, which tend to expand abroad, cross board of nations to gain more resources and profits so as to be called transnational corporation. In an easy model of supply chain, companies exploit resources, then manufacture goods, sell goods or provide services to consumers next, and finally gain profit so as to expand their entities and get more profit. It is the way that companies survive and be living or viable. Human society lies within billions of the economic chains that combine to form tremendous networks, together with its economic activities.

Economic activities exist in human society and depend deeply on human behavior, such as trade, transaction, and communication. It is human who makes the society of each nation living, specific and viable. Meanwhile there is also a kind of substance which exists in billions of organisms commonly, of which entire global ecological system is composed together with inorganic factors.

Organism is any living structure capable of growth and reproduction. What makes life different is that organisms are made from three basic classes of molecule: amino acids, carbohydrates and lipids, which are vital for life. But the 
core factors of life are electrons within these biochemical molecules, making physiological reaction impossible, composing bioactive structure and performing biochemical function [6].

Human-electron analogy exists in several points:

Both are the basic factors and substances of the organization and widely distributed. In spite of different colors, forms, structures or behaviors for every species of organism, or religions, cultures, classes or regime for human nation and society, they are the core substantial ones.

Both are active and "liquid", willing to move and make change. Chemical reaction occurs because of electronic transition, and history is driven by human choices; human migration promotes progress of human civilization.

Both are responsible for organization viable and living. Electron flow implies energy change which is a characteristic of living organism, and city would be abandoned or desolation and ruin without human, even a civilization shall decline and fall with fewer human population.

For these reasons, VSM model derived from human nervous system can be introduced to transnational management. Electron implies the importance of biochemistry for the research of physiology, just as human implies management for the one of corporation business administration.

\section{VSM for Transnational Management}

Transnational corporation is faced with changeable and complicated environment in the Internet age. Global Competitors as change agents, force for local differentiation and responsiveness, strict government demands, variable cultural difference, growing pressures for localization and forces for worldwide innovation and learning together with information explosion make companies difficult to survive and maintain living. So the structural organization should be changed not only to fit their specific environment but also to make strategic decisions coordinately, which is like autonomy but still integrated network as human physiological structure.

VSM for Transnational Management is plotted in Figure 1 and elaborated below:

(1) System 1 in a viable system contains several primary activities, like organs for human. Each System 1 primary activity is itself a viable system due to the recursive nature of systems, concerned with performing a function that implements at least part of the key transformation of the organization.

In a transnational corporation, system 1 means subsidiaries all over the world, with a viable system of each, dealing with host nation's government, 
acquiring information and knowledge in specific environment. It acts not only an efficient sensor, but also a cultural interpreter able to communicate, and frontline implementer of corporate strategy.

Fig.1 VSM model for transnational corporation

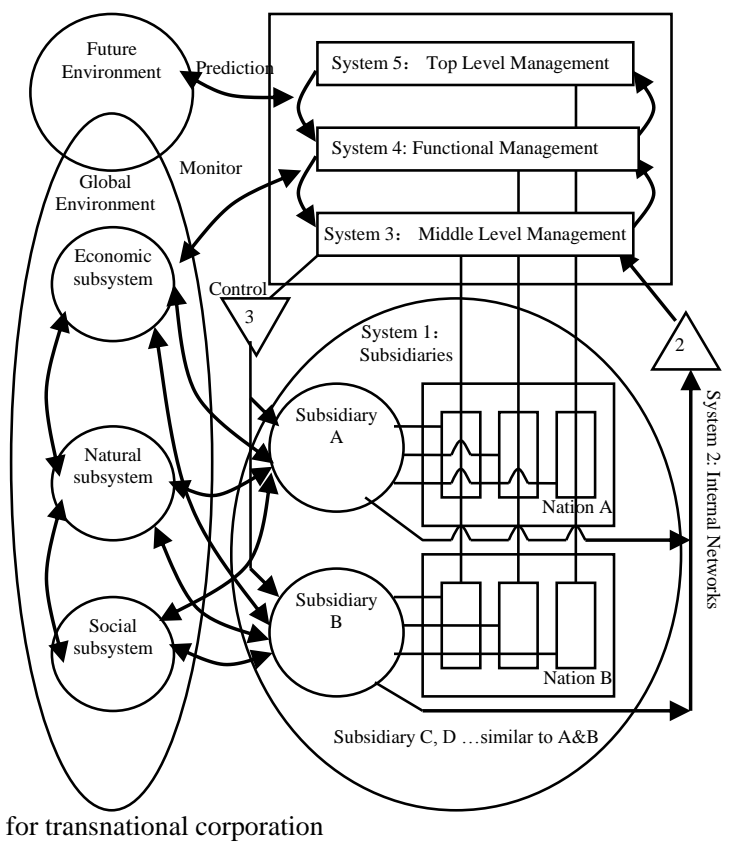

(2) System 2 represents the information channels and bodies that allow the primary activities in System 1 to communicate with each other and which allow System 3 to monitor and co-ordinate the activities within System 1, representing the scheduling function of shared resources to be used by System 1, like sympathetic nerves for human.

In a transnational corporation, system 2 means effective internal networks providing quick information communication platform. In the Internet age, it shall be easy by establishing workgroup using instant messenger clients or traditional Bulletin Board System. However, information sharing would be critical considering time zone, hierarchy and bureaucracy.

(3) System 3 represents the structures and controls that are put into place to establish the rules, resources, rights and responsibilities of System 1 and to provide an interface with Systems 4 and 5, representing the big picture view of the processes inside of System 1, like sympathetic nerves for human.

In a transnational corporation, system 3 means global business managers or middle level management. As a global business strategist, architect of asset and 
resource should be configured, the information daily provided by system 2 be treated and cross boarder problems be coordinated and solved.

(4) System 4 is responsible for looking outwards to the environment to monitor how the organization needs to adapt to remain viable, like spinal cord for human.

In a transnational corporation, system 4 means worldwide functional management, or the intelligence department. As a worldwide intelligence scanner, it should be smart and farsighted, linked effectively with system 2 and 3 through informal networks and maintained through frequent meetings, visits, travels and transfers, identifying where best practices are being developed and implemented, so as to lead transnational innovation.

(5) System 5 is responsible for policy decisions within the organization as a whole to balance demands from different parts of the organization and steer the organization as a whole.

In VSM model compared with human physiology, system 5 is like brain. In a transnational corporation, system 5 means top level corporate management, providing direction and purpose, leveraging corporate performance, ensuring continual knowledge renewal. It is crucial for a company's survival.

\section{Analysis and Discussion}

In the Internet age, the world economy is affected by many uncertainty factors. Most transnational corporations survive and live in an ecological environment composed of several subsystems like economic one as currency, exchange rate, while natural one as resource scarcity and allocation, changeable weather and extreme climate, also social and administrative one as culture, behavior, government demands, regulation and laws. Entire global environment consists of different cross-board subsystems, which is strong force for local differentiation and responsiveness, and corporation as a whole shall respond to diverse forces simultaneously so worldwide innovation and learning would be a tough job.

In the model above, system 1 is composed of basic subsidiaries located in different nations and will fit the pressure of localization. System 2 and 3 provide a dual-direction control for information, while 2 stresses on information seeking, acquiring, dissemination and 3 on information processing, classification, coordination. System 2 makes a positive impact for push and integration, while system 3 makes some negative one for differentiation and characteristic, more coordination. As middle management a transition one, System 1, 2 and 3 make contact with national localization directly (biggest circle in Fig.1) and respond to current global environment, while system 3, 4 and 5 make preparation and 
decision for future one, constructing a intellectual flexible organization(square above circle in Fig.1).

\section{Conclusion}

VSM model is a viable system organized in such a biologically nervous system way as to meet the demands of surviving in the changing environment. It is introduced here to illustrate a strategic organizational model for modern company, especially for transnational corporation faced with tough international relationship and differentiation localization, also huge amount of information together with more media tools making it fit the global economy and ecological environment. Human-electron analogy makes a transitional bridge for management and physiology to classify concept of two fields. Maybe more cases and data would be needed to demonstrate or implement the model, so there is still some more work to do.

\section{References}

1. J. M. Stopford and L. T. Wells. Managing the multinational enterprise: Organization of the firm and ownership of the subsidiaries. Vol. 2. (Basic Books, New York, 1972).

2. C. A. Bartlett and S. Ghoshal. Matrix management: not a structure, a frame of mind. Harvard Business Review. 68(4), pp. 138-145, (1990).

3. C. A. Bartlett and P. W. Beamish. Transnational Management: Text, Cases and Readings in Cross-border Management, $7^{\text {th }}$ edn. (McGraw Hill Education, 2014).

4. S. Beer. Brain of the Firm. (The Penguin Press, London, Herder and Herder, USA, 1972).

5. S. Beer. Diagnosing the System for Organizations. (John Wiley, London and New York, 1985).

6. Kun Shi. Eco-city: A living organism system. Advanced Materials Research, Vols. 616-618, pp. 1280-1284, (2013). 